\title{
AC 2008-1917: DEVELOPMENT OF ELECTRIC MACHINE LIVE COMPUTATIONAL MODULES FOR CLASSROOM USE
}

Clayton Hochstrasser, The Ohio State Univ.

Joseph Werner, The Ohio State Univ.

Donald Kasten, Ohio State University 


\title{
Development of Electric Machine Live Computational Modules for Classroom Use
}

\begin{abstract}
Live computational and graphical capability in engineering lecture classes can provide an effective tool to illustrate cause-and-effect relationships. The computational process must not over-shadow the concept to be illustrated, but must provide an easy means to clearly demonstrate results of the calculations. Visual Basic was used to develop calculation modules that would be very portable within the current classroom IT capabilities. The course that was used as the foundation for this development was an undergraduate course in electric machines and power systems. This paper will discuss the rationale motivating the effort, illustrate the capabilities of the software and report on the response of the students to the use of this as a teaching tool.
\end{abstract}

\section{Rationale}

Many times in the electrical engineering lecture classes, the derivation of equations and subsequent use of these derived equations to illustrate cause-and-effect relationships are somewhat limited in the class-room environment due to the calculation overhead. For example, in the power systems area of electrical engineering, consider the per phase equivalent circuit of a three-phase induction machine. Having developed the equivalent circuit, it is rather mathematically tedious to determine the effect on the input quantities (e.g., current and power) as a function of motor speed or horse-power loading. Certainly simplifications can be made to expedite the calculations; however in some cases these simplifications may limit the realism of the result. By developing some digital interactive modules, these kinds of cause-and-effect relationships can be demonstrated during lecture using the facilities currently available in most classrooms. This will require interactive capability, calculating capability, as well as graphing functionality. Thus, rather than just illustrating the mechanics of the solution for a specific operating point, a number of operating points can be calculated and results displayed in an organized fashion. Tools such as this can make the instructional process investigative in nature, by addressing what-if scenarios. Visual Basic was chosen as the software to do this development work.

\section{Visual learning}

"Visual learning is an important method for exploiting students' visual senses to enhance learning and engage their interest." ${ }^{1}$ Though this reference focuses on underrepresented students in the technical fields, the concepts are applicable across the range of the student population. Ref. 1 was part of a special issue of the IEEE Computer Graphics and Applications society on "innovative ways in which people were teaching computer graphics and ways in which people were using computer graphics to teach other topics.", The authors of a paper entitled "Learning About Fields and Waves Using Visual Electromagnetics" developed both stored-solution mode and generated-solution mode computer graphics to attract students into the field of electromagnetics. ${ }^{3}$ Their generated-solution mode is similar to the live computational modules as described in this paper. Interactive visual activities were utilized for teaching engineering 
students technical Japanese. ${ }^{4}$ In this case the tools were developed for student use; whereas the tools developed in the project described in this paper are primarily for instructor use during lectures to enhancing learning.

The efforts presented here in this paper represent undergraduate students and faculty of an electrical engineering program in a state university. Students always ask for more examples to illustrate the concepts presented in the classroom lectures. Whether their motivation for more examples is to just learn the mechanics of the problems without really understanding the principles involved or that the examples do reinforce their understanding of those principles, the fact remains, at least for these authors, that there is a need to illustrate numerical examples in the classroom.

\section{Organization of effort}

At our university, the College of Engineering has made funds available through small grants for utilizing technologies to enhance teaching and learning. The effort described here was initiated for a first course in the area of power systems and energy conversion for undergraduates. This course follows a course in circuit analysis. The specific topics for the calculation modules thought to be most useful would be for illustration of phasors as related to a 3-phase system, transformer circuit modeling and circuit analysis, and rotating machine (DC, 3-phase AC induction and synchrous) modeling and analysis.

Undergraduate students who have recently taken this class are the primary participants in the design and development of the modules. The perspective they bring to the effort is very important so that the modules will be designed from the view-point of the student, and how they learn, rather than that of the lecturer, though obviously there is communication between the faculty and students in this development process.

As modules are developed and ready for testing, they will be used in the classroom. Though this particular class is taught every term during the year, the faculty associated with this project do not teach it each term of every year. The modules will be made available to all who teach this course, or related courses, that wish to use them. Though these modules are only used during lecturers, and not available for student use during exams, student achievement on certain quiz and exam questions that are directly related to developed modules, will be compared with student achievement prior to the use of these modules. Course evaluation questionnaires administered at the end of the term will address the use of the modules.

\section{Choice of software}

The most important feature for any software used, is that the developed program must be transportable. Many of our classrooms have a PC driven projector system with additional A/V capabilities; the PC is also connected to the Internet either wirelessly or hard wired. Visual Basic (VB) was chosen for use because of university licensing availability on campus and its relative independence of machine and operating system platform. There are some draw-backs to VB, two of which are identified here. One is that VB does not have any built-in functionality for complex mathematics as would be used in power system circuit analysis applications. That 
however is not that much of an issue as various complex functions can be identified, for example, complex addition, complex multiplication, and converting from rectangular representation to polar representation and vice versa. The second draw-back is related to graphing; VB does have elementary draw functions, rotate functions, etc., but as far as graphing an equation or equations, that feature must be programmed. A share-ware graphing routine was found and is implemented ${ }^{5}$; its features will be illustrated later in examples.

\section{Examples}

The examples presented in this paper were chosen to illustrate general capabilities of the modules and do not necessarily represent all modules, nor the order, needed in the logical progression of topics as presented in the course. However, at each step in the learning process for the course there will be appropriate modules consistent with the level of topics being covered at that time. Since students who take this course must have successfully taken a circuit's course, they are familiar with circuit analysis techniques and basic principles such as Ohm's law and Kirchhoff's laws.

All modules have a number of common sub modules. Some of these common modules would include complex math functions and graphing functions.

The first example presented is that of a simple series circuit illustrating a load, where the applied voltage and circuit impedance can be chosen; refer to Figure 1. The resulting current is displayed and the choice to draw a phasor diagram of the applied voltage and current is provided. It is felt that familiarity with phasor diagrams can significantly enhance the understanding of the concept of lead and lag specifications with regard to power factor.

Modules such as that illustrated in Fig. 1 which cover topics introduced in previous courses are designed to reinforce current knowledge and set the stage for extending the concepts to this course. In other cases, as will be illustrated by later examples, the purpose is to illustrate new topics, so the lecture narrative that goes along with the use of these modules will have a different approach.

Figure 1 illustrates several standard type features that will be included in most all modules. One is the choice of entering the system variables and parameters that are complex quantities in either rectangular or polar form as is shown by the selection in the bottom right-hand corner of the screen. Once the data has been entered, the solve button is used to initiate the specific calculation; in the case of the example of Fig. 1 it would be to calculate the current, also a complex number. This example is simply an application of Ohm's Law and displaying the answer either in rectangular or polar form as initial chosen. A second standard feature is the ability to then draw a phasor diagram of the voltage and current using the plot button. 


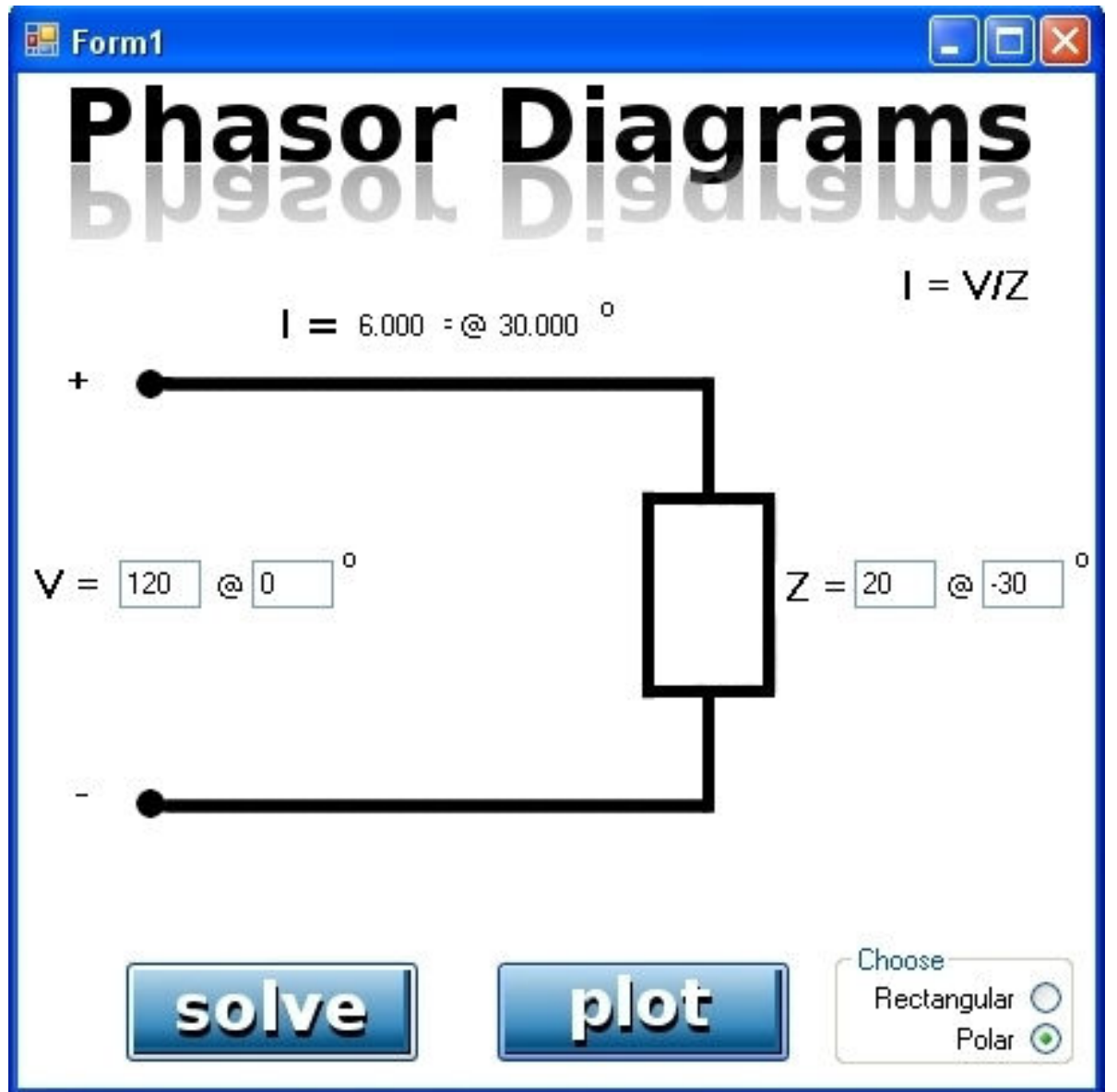

Figure 1: Example of a simple load circuit.

Figure 2 shows the screen that would result if the plot button had been selected for the example of Fig. 1; it is a complex plane on which the phasors are drawn. The imaginary and real axes of the complex plane are labeled as "i" and "r," respectively. If the two phasors are of significantly differing magnitudes, then there is an automatic scale factor applied to the smaller, as indicated in the bottom left hand part of the screen.

A second example is illustrated in Fig. 3 for a 1-phase transformer. The circuit model shown keeps the primary winding impedance and secondary winding impedance separated, rather than combined as many times might be the case. The transformer parameters are input quantities and for this example, the transformer secondary loading is specified. The purpose of this module is to calculate the required primary voltage to support the specified load conditions. The calculated values of various voltage drops and currents within the circuit are also shown in the figure. 


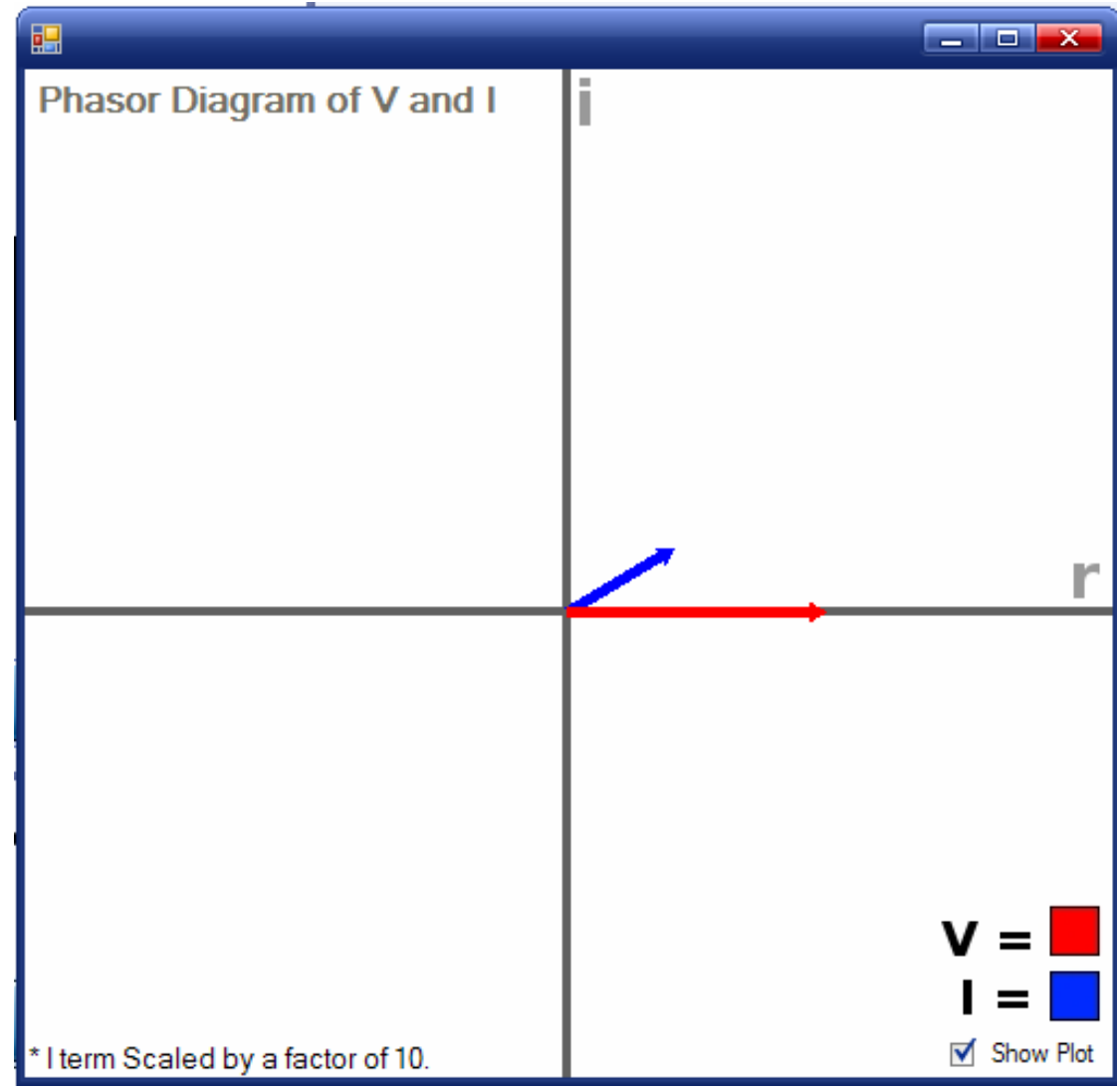

Figure 2: Phasor diagram for example of Figure 1.

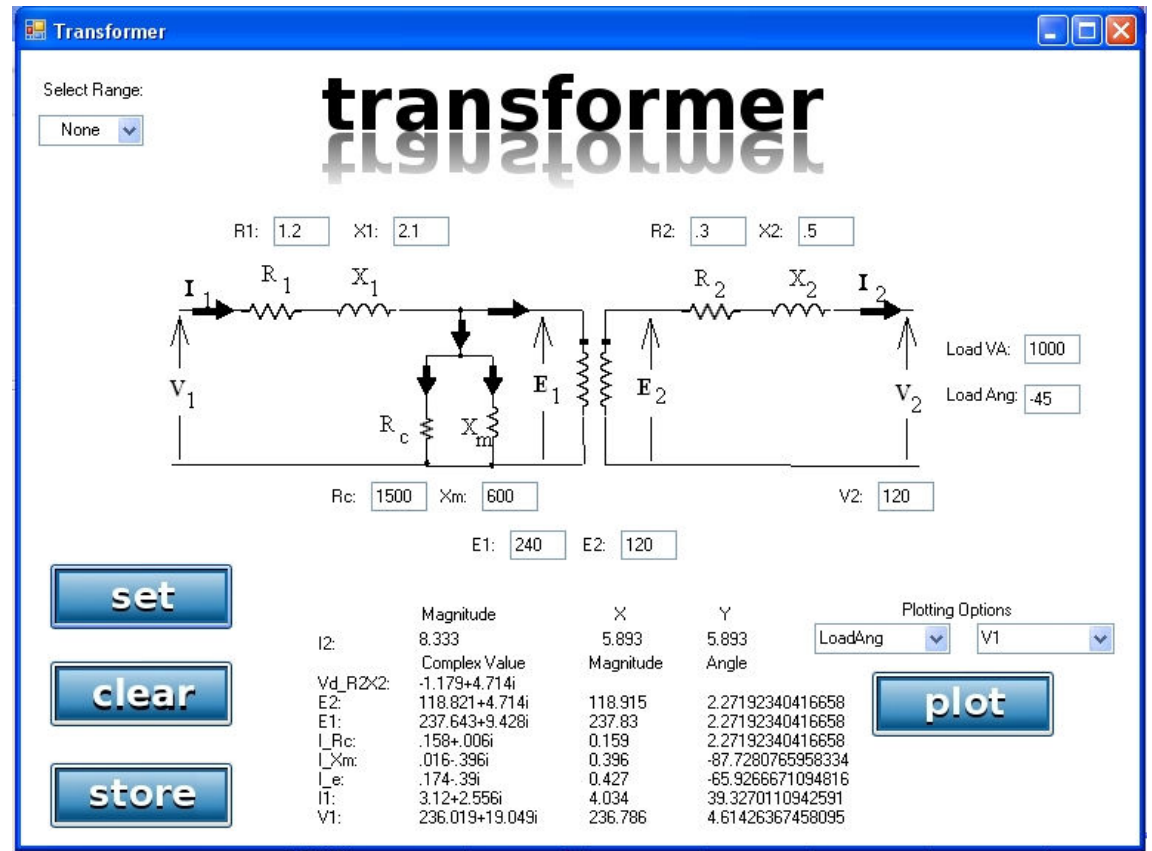

Figure 3: Example of single-phase transformer model and calculations. 
One of the initial driving motivations for the effort associated with this paper was to provide not only live calculation ability for a single operating point defined by the user, but also to illustrate such calculations for a range of values for a chosen variable. The idea of illustrating how the system variables change as a function of changes with another system variable or system parameter, i.e., cause-and-effect relationships, was felt to be an important learning concept. For example, by maintaining a constant load voltage and constant load VAs, and varying the angle of the load VAs, the required primary voltage for the circuit of Fig. 3 changes. This is illustrated in Fig. 4 which gives a plot of primary voltage for the transformer of Fig. 3 for this condition as a function of the load VA angle. Thus visually, backed up with the appropriate calculations, the students can observe this cause-and effect relationship for the required source voltage as it relates to load power factor. This can be used to introduce the concept of power factor correction capacitors.

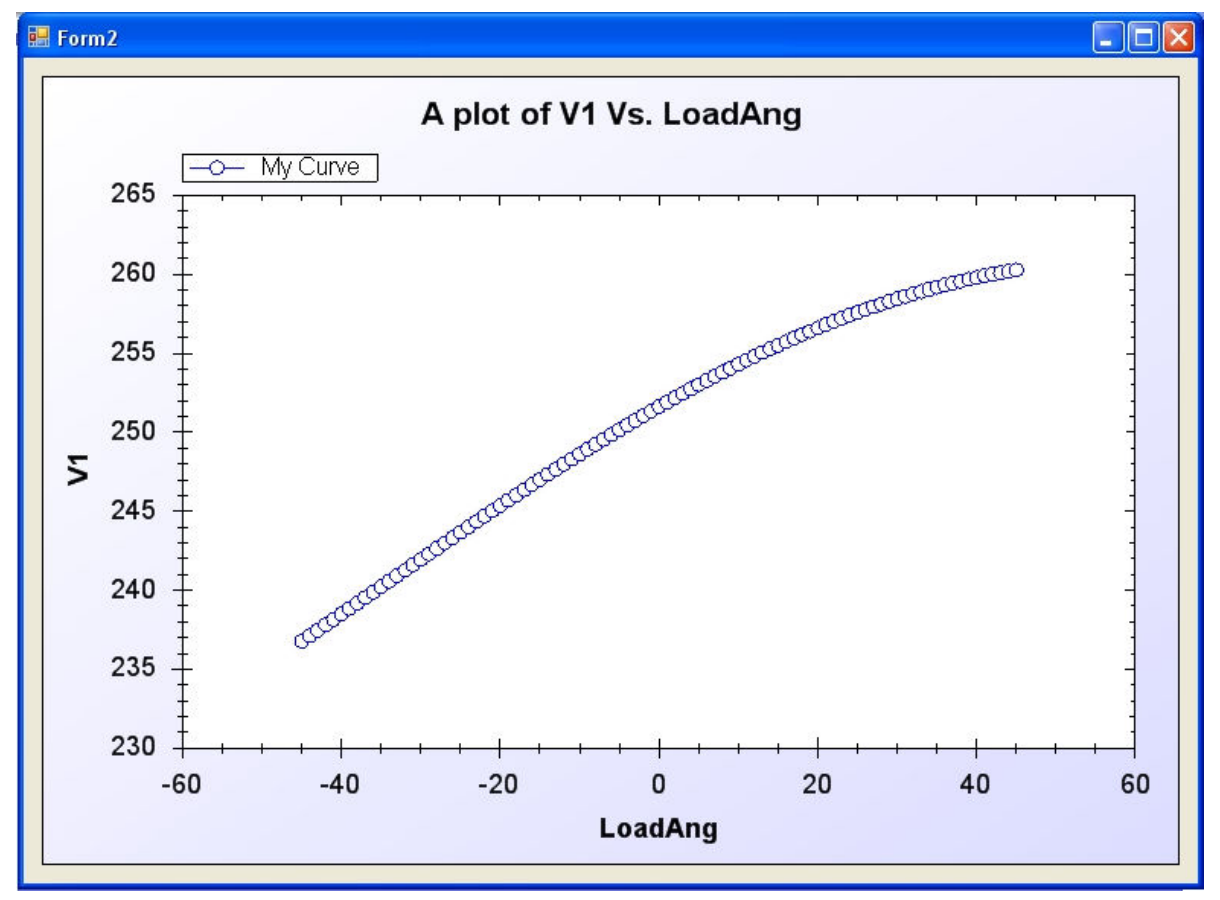

Figure 4: Single-phase transformer model of Fig. 3 illustrating the use of graphing results for a range of a chosen variable -- primary voltage vs. the load VA angle.

A third example is shown in Fig. 5. It represents the per phase equivalent circuit of a 3-phase synchronous machine. This screen can be used for either motor or generator action, and the appropriate Kirchoff's voltage law equation is shown. The machine parameters, armature resistance, $R_{a}$, and synchronous reactance, $X_{s}$, must be entered. Then the user has a choice of what calculation is desired; that is, given the terminal voltage, $\mathrm{V}_{\mathrm{a}}$, and the armature current, $\mathrm{I}_{\mathrm{a}}$, with the associated power factor, calculate the required emf, $\mathrm{E}_{\mathrm{a}}$; or given $\mathrm{E}_{\mathrm{a}}$ and the current and calculate the terminal voltage. The choice of which calculation desired is given in the upper left hand corner of the screen by the motor-generator choice. Also the choice between using rectangular or polar form of the complex variables is provided; in Fig. 5 polar form is chosen. 


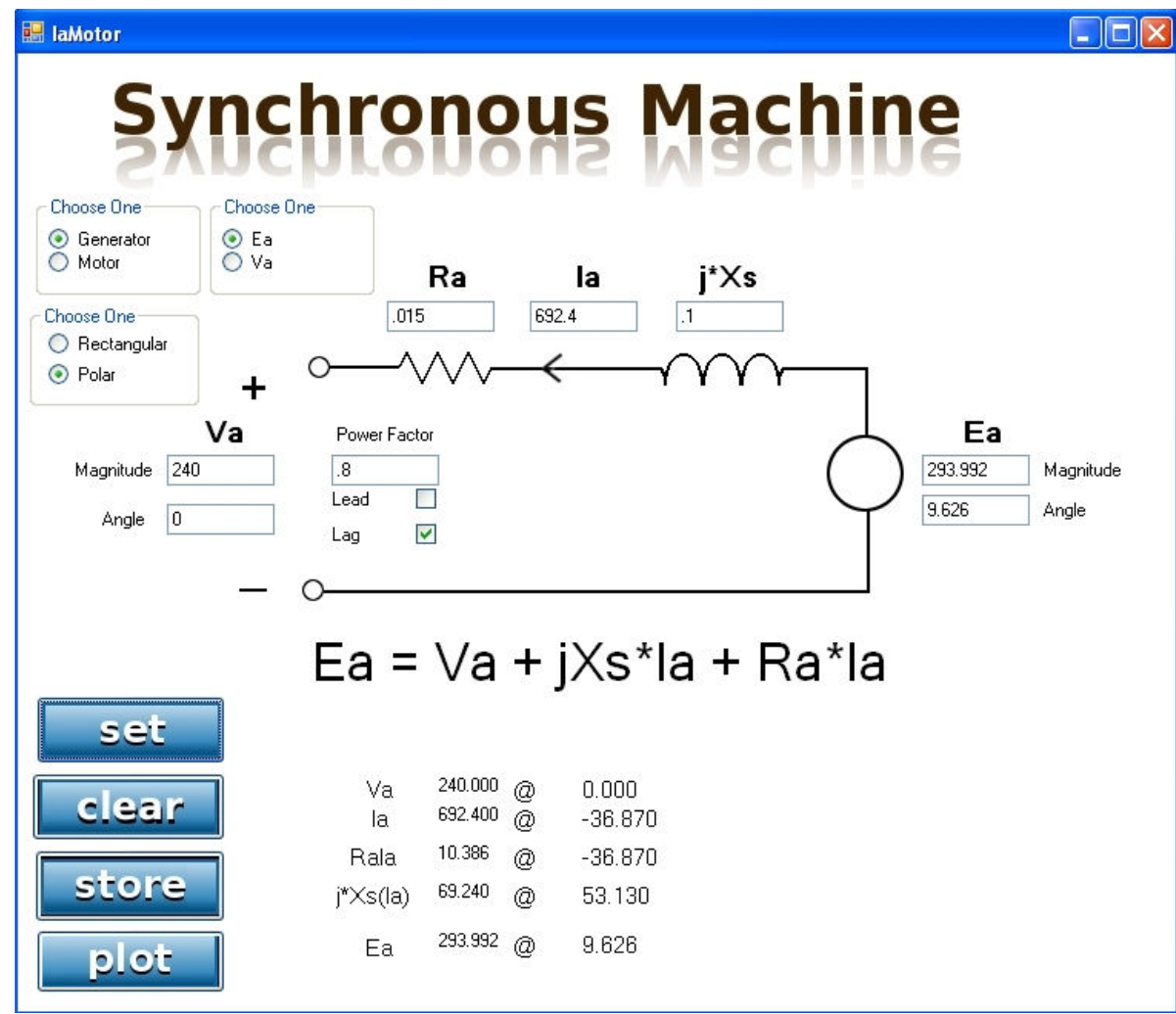

Figure 5: Example illustrating synchrous machine calculations.

Additional modules and features to be implemented

With this particular course, there is the potential for many other calculation modules. Converting from time domain functions, e.g., $\mathrm{v}(\mathrm{t})=\mathrm{V}_{\max } \sin (\omega \mathrm{t}+\theta)$, to a frequency domain representation, i.e., a phasor, may not have been emphasized that much in previous courses, and a module to show a number of examples of a time domain function and its corresponding phasor representation, or vice versa, can be very beneficial. Also, illustrating the time domain and corresponding phasor relations for a balanced 3-phase set of voltages and currents helps significantly in the understanding of 3-phase systems.

Transformer analysis as illustrated with Fig. 3 and 4 for what is essentially a voltage regulation type problem is typical also for this course. Efficiency and the effect of "away from the norm" values for certain parameters in the circuit model can easily be investigated.

Figure 5 illustrates an application for rotating machines; however there are many more applications. The torque-speed curve for a 3-phase induction machine and V-curves for a 3phase synchronous motor are classic examples of typical calculations for this course. The use of phasor diagrams for these machines and also the plotting for a range of values, as illustrated with Fig. 4, will be very useful. 


\section{Disclaimer}

The purpose of the proposed project is not to develop a stand-alone on-line course. It does not, nor is it intended to, provide all the necessary background nor all the narrative necessary to justify the models and to interpret the results from such examples as that illustrated above. Its purpose is to provide analytical tools whereby practical illustrative numerical examples can be used in the classroom environment to show "cause-and-effect" relationships, hence, lead into design criteria if desired; its purpose is to complement the lectures, not replace.

Also, one should not over-use these modules in place of lectures on the associated theory. There is a balance between the "straight lecture" and the illustration of concepts with examples, and that varies with the course and with the professor, one-size does not necessarily fit all. As stated above, the purpose of this calculation ability is to supplement, not replace the traditional lectures.

\section{Observations to date}

The level of effort in this software development is significant. Student participation has been and continues to be excellent (spoken on behalf of the faculty author). The self learning and the organizational and communication skill development of the student programmers is an excellent side benefit. Limited classroom use of the modules has been made to date as it is detrimental to student learning to utilize tools that are not mature in their development.

\section{Future goals}

As indicated in the Abstract, the objective of this effort is to enhance teaching and learning with the use of live-computational graphics in the classroom for a first course in power systems and energy conversion. A number of calculation modules using Visual Basic have been defined and are in various stages of development. There has been limited exposure in the class room with the use of these modules to date because of the time-table for this project. The major thrust in the coming terms will be to use the modules in the classroom, obtain the candid comments of the students and modify accordingly. Direct student feedback in the form of comments and also evaluation of student success on various topics can be measured compared with before these modules were available.

\section{Acknowledgements}

The authors would like to acknowledge The Ohio State University College of Engineering for their financial support through the Utilizing Technologies to Enhance Teaching and Learning grant program for academic year 2007-2008. 


\section{Bibliography}

1. McGrath, M.B., Brown, J.R., "Visual Learning for Science and Engineering," IEEE Trans. on Computer Graphics and Applications, Vol. 25, Issue 5, Sept.-Oct. 2005, pp. 56-63.

2. Bailey, M., Cunningham, S., "Guest Editors' Introduction: Computer Graphics in Education,” IEEE Trans. on Computer Graphics and Applications, Vol. 25, Issue 5, Sept.-Oct. 2005, pp. 23-23.

3. Cole, R.W., Miller, E.K., Chakrabarti, S., Gogineni, S., "Learning About Fields and Waves Using Visual Electromagnetics," IEEE Trans. on Education, Vol. 33, No. 1, Feb. 1990, pp. 81-87.

4. Bhattacharya, Y., Bhattacharya, M., "Work in Progress: Design of a Visual Learning Tool for Japanease," $36^{\text {th }}$ ASEE/IEEE Frontiers in Education Conf., Oct. 18-31, 2006, San Diego, CA, paper M3F-15.

5. http://www.zedgraph.org 